\title{
TIME-ORDERED OPERATORS. II
}

\author{
BY
}

TEPPER L. GILL ${ }^{1}$

\begin{abstract}
In this paper, we substantially improve on the work of [G1]. After constructing the general mathematical foundations for linear time-ordered evolution equations, we apply our results to show that both the perturbation expansion and the Feynman diagram method are mathematically sound. We provide a remainder term so that the expansion may be considered exact at all orders. We then show that time-ordered operators naturally induce an operator-valued path integral whenever a transition kernel is given.
\end{abstract}

Introduction. This is a natural extension of the program begun in [G1]. In this paper we make a number of substantial improvements in the work reported there. In $\S \S 1$ and 2, we construct the mathematical foundations for the general theory of time-ordered linear evolution equations. In $\$ 3$ we show that the time-ordered evolution operator provides a natural approach to generalized additivity of unbounded operators. We show that the evolution operator for the sum of two generators of contraction semigroups exists almost everywhere without imposing any domain conditions, and we prove a number of general theorems of the perturbation expansion type. We also show that whenever a transition kernel exists, the timeordered evolution operator naturally induces an operator-valued path integral.

Preliminaries. As in [G1], we assume that $\alpha$ is any uniform faithful reasonable norm on $\otimes_{\tau} B(\tau)$, where $B(\tau)$ is a separable Banach space for each $\tau \in[0, T]$, $T>0$. The closure of $\otimes B(\tau)$ with respect to $\alpha$ is a Banach space of type $v$, and we denote it by $\otimes_{\tau}^{\alpha} B(\tau)$. In order to simplify our work, we assume $B(\tau)=B$ for each $\tau$. The general case will cause no great problem.

We assume that unless otherwise stated, all operators of the form $\tilde{A}(s)$ are strongly continuous operators in the conventional sense, while those of the form $A(s)$ are time-ordered. In either case we always assume that they are infinitesimal generators of contraction semigroups at each point in time. $\tilde{D}(s)$ is the domain of $\tilde{A}(s)$ while $D(s)$ is the domain of $A(s) . D=\otimes_{s}^{\alpha} D(s)$.

Let $\left\{Q_{m} \mid m \in \mathbf{N}\right\}$ be a sequence of partitions of $[0, T]$ such that $\lim _{m \rightarrow \infty}\left|Q_{m}\right|=0$, (mesh) set $\mathbf{P}_{n}=[0, t) \cap Q_{m}$, where $n$ is the number of partition points of $[0, t]$ in $Q_{m}$ with $0<t<T$. This is done for later convenience. We assume that for each $n \in \mathbf{N}$, a $\mathbf{P}_{n}$ exists, and we will use one distinct, fixed set $\left\{\mathbf{P}_{n} \mid n \in \mathbf{N}\right\}$.

Received by the editors March 15, 1982 and, in revised form, September 20, 1982.

1980 Mathematics Subject Classification. Primary 47D05, 35K22, 81C12; Secondary 81B99, 34K30.

${ }^{1}$ On leave from the Department of Mathematics, Howard University, Washington D.C. 20059. 
The Riemann-complete integral is not used in order to simplify the presentation. It is clear what the appropriate generalization should be.

1. Let

$$
A^{z}(\tau)=\frac{\exp \{z A(\tau)\}-I}{z}, \quad z>0,
$$

and recall that $A^{z}(\tau)$ is a bounded linear operator defined on $B(\tau)$ for each $\tau$ and $s-\lim _{z \downarrow 0} A^{z}(\tau)=A(\tau)$. Set

$$
Q_{n}^{z}[t, 0]=\sum_{j=1}^{n} \Delta t_{j} A^{z}\left(\tau_{j}\right), \quad \bar{Q}_{n}^{z}[t, 0]=\sum_{j=1}^{n} \int_{t_{j-1}}^{t_{j}} d \tau E\left[\tau_{j}, \tau\right] A^{z}(\tau) .
$$

Define $Q_{\lambda}^{z}[t, 0]$ and $\bar{Q}_{\lambda}^{z}[t, 0]$ by

$$
\left\{\begin{array}{l}
Q_{\lambda}^{z}[t, 0]=e^{-\lambda T} \sum_{n=0}^{\infty} \frac{(\lambda T)^{n}}{n !} Q_{n}^{z}[t, 0], \\
\bar{Q}_{\lambda}^{z}[t, 0]=e^{-\lambda T} \sum_{n=0}^{\infty} \frac{(\lambda T)^{n}}{n !} \bar{Q}_{n}^{z}[t, 0],
\end{array}\right.
$$

THEOREM 1.1. (1) $\lim _{\lambda \rightarrow \infty} Q_{\lambda}^{z}[t, 0]=\lim _{\lambda \rightarrow \infty} \bar{Q}_{\lambda}^{z}[t, 0]=Q^{z}[t, 0]$ exists and $Q^{z}[t, 0]$ $=Q^{z}[t, s]+Q^{z}[s, 0], 0 \leqslant s<t$.

(2) $s$ - $\lim _{z \downarrow} Q^{z}[t, 0]=Q[t, 0]$ exists, is densely defined, and $Q[t, 0]=Q[t, s]+$ $Q[s, t]$.

Proof. First let us note that from (1.0) we have $\left\|A^{z}(\tau)\right\| \leqslant 2 / z$, from which it follows that $\left\|\bar{Q}_{n}^{z}[t, 0]\right\| \leqslant 2 t / z$, and the same for $Q_{n}^{z}[t, 0]$ independent of $n$. Using this, it is easy to see that $\max \left(\left\|Q_{\lambda}^{z}[t, 0]\right\|,\left\|\bar{Q}_{\lambda}^{z}[t, 0]\right\|\right) \leqslant 2 t / z$ independently of $\lambda$, so that $Q_{\lambda}^{z}[t, 0]$ and $\bar{Q}_{\lambda}^{z}[t, 0]$ are bounded linear operators.

Proof of (1). Let $\lambda_{1}$ and $\lambda_{2}$ be arbitrary. Then

$$
\left\|Q_{\lambda_{1}}^{z}[t, 0]-Q_{\lambda_{2}}^{z}[t, 0]\right\| \leqslant \frac{2 t}{z} \sum_{n=0}^{\infty} \frac{T^{n}}{n !}\left|\lambda_{1}^{n} e^{-\lambda_{1} T}-\lambda_{2}^{n} e^{-\lambda_{2} T}\right|
$$

since $\left|\lambda_{1}^{n} e^{-\lambda_{1} T}-\lambda_{2}^{n} e^{-\lambda_{2} T}\right| \rightarrow 0$ as $\lambda_{1}, \lambda_{2} \rightarrow \infty$ for each $n$, and we see that the family $\left\{Q_{\lambda}^{z}[t, 0] \mid \lambda>0\right\}$ is a Cauchy sequence and hence has a unique limit. The same is true for the family $\left\{\bar{Q}_{\lambda}^{z}[t, 0] \mid \lambda>0\right\}$. In order to show that these two limits are identical, note that $c$-continuity of the family $\{A(t) \mid t \in[0, T]\}$ implies the same for $\left\{A^{z}(t) \mid t \in[0, T]\right\}, z>0$.

$$
\left\|\bar{Q}_{\lambda}^{z}[t, 0]-Q_{\lambda}^{z}[t, 0]\right\| \leqslant e^{-\lambda T} \sum_{n=0}^{\infty} \frac{(\lambda T)^{n}}{n !} \sum_{j=1}^{n} \int_{t_{j-1}}^{t_{j}} d \tau\left\|E\left[\tau_{j}, \tau\right] A^{z}(\tau)-A^{z}\left(\tau_{j}\right)\right\| .
$$

c-continuity on $[0, T]$ implies $c$-uniform continuity, hence given $\varepsilon>0, \exists \delta>0$ such that $\Delta t_{j}<\delta \Rightarrow\left\|E\left[\tau_{j}, \tau\right] A^{z}(\tau)-A^{z}\left(\tau_{j}\right)\right\|<\varepsilon / t, \tau \in\left[t_{j-1}, t_{j}\right)$. Since $\left|P_{n}\right| \rightarrow 0, \exists N \exists$ $n \geqslant N \Rightarrow \Delta t_{j}<\delta \forall 1 \leqslant j \leqslant n$; hence 


$$
\begin{aligned}
& \left\|\bar{Q}_{\lambda}^{z}[t, 0]-Q_{\lambda}^{z}[t, 0]\right\| \leqslant \sum_{n=0}^{N} e^{-\lambda T} \frac{(\lambda T)^{n}}{n !} \sum_{j=1}^{n} \int_{t_{j-1}}^{t_{j}} d t\left\|E\left[\tau_{j}, \tau\right] A^{z}(\tau)-A^{z}\left(\tau_{j}\right)\right\| \\
& +\sum_{n=N+1}^{\infty} e^{-\lambda T} \frac{(\lambda T)^{n}}{n !} \sum_{j=1}^{n} \int_{t_{j-1}}^{t_{j}} d \tau(\varepsilon / t) \\
& \leqslant \sum_{n=0}^{N} e^{-\lambda T} \frac{(\lambda T)^{n}}{n !} \sum_{j=1}^{n} \int_{t_{j-1}}^{t_{j}} d t\left\|E\left[\tau_{j}, \tau\right] A^{z}(\tau)-A^{z}\left(\tau_{j}\right)\right\|+\varepsilon .
\end{aligned}
$$

Let

$$
\lambda \rightarrow \infty \Rightarrow \lim _{\lambda \rightarrow \infty}\left\|\bar{Q}^{z}[t, 0]-Q_{\lambda}^{z}[t, 0]\right\|=\left\|\bar{Q}^{z}[t, 0]-Q^{z}[t, 0]\right\| \leqslant \varepsilon .
$$

As $\varepsilon$ is arbitrary we see that $\bar{Q}^{z}[t, 0]=Q^{z}[t, 0]$. Let $0 \leqslant s \leqslant t$ and let $k=k(s)$ be such that $t_{k+1}>s \geqslant t_{k}, 1 \leqslant k<n$. For each $n$, set

$$
Q_{n}^{z}[t, s]=\sum_{j=k}^{n} \Delta t_{j} A^{z}\left(\tau_{j}\right), \quad Q_{n}^{z}[s, 0]=\sum_{j=1}^{k-1} \Delta t_{j} A^{z}\left(\tau_{j}\right) .
$$

Then

$$
\begin{aligned}
Q_{\lambda}^{z}[t, 0] & =\sum_{n=0}^{\infty} e^{-\lambda T} \frac{(\lambda T)^{n}}{n !}\left(Q_{n}^{z}[t, s]+Q_{n}^{z}[s, 0]\right) \\
& =Q_{\lambda}^{z}[t, s]+Q_{\lambda}^{z}[s, 0] \Rightarrow Q^{z}[t, 0]=Q^{z}[t, s]+Q^{z}[s, 0] .
\end{aligned}
$$

Proof of (2). The proof of (2) is similar to that of Theorem 4.3 in [G1] and hence will only be outlined. Let $\phi \in D$. Then

$$
\begin{aligned}
\| Q^{z_{1}}[t, 0] \phi & -Q^{z_{2}}[t, 0] \phi\|\leqslant\| Q^{z_{1}}[t, 0] \phi-Q_{\lambda}^{z_{1}}[t, 0] \phi \| \\
& +\left\|Q_{\lambda}^{z_{2}}[t, 0] \phi-Q^{z_{2}}[t, 0] \phi\right\|+\left\|Q_{\lambda}^{z_{1}}[t, 0] \phi-Q_{\lambda}^{z_{2}}[t, 0] \phi\right\| .
\end{aligned}
$$

Choose $\lambda_{1}$ such that

$$
\lambda \geqslant \lambda_{1} \Rightarrow\left\|Q^{z_{1}}[t, 0] \phi-Q_{\lambda}^{z_{1}}[t, 0] \phi\right\|<\varepsilon / 3,
$$

and $\lambda_{2}$ such that

$$
\lambda \leqslant \lambda_{2} \Rightarrow\left\|Q^{z_{2}}[t, 0] \phi-Q_{\lambda}^{z_{2}}[t, 0] \phi\right\|<\varepsilon / 3,
$$

$$
\left\|Q_{\lambda}^{z_{1}}[t, 0] \phi-Q_{\lambda}^{z_{2}}[t, 0] \phi\right\| \leqslant e^{-\lambda T} \sum_{n=0}^{\infty} \frac{(\lambda T)^{n}}{n !} \sum_{j=1}^{n} \Delta t_{j}\left\|A^{z_{1}}\left(\tau_{j}\right) \phi-A^{z_{2}}\left(\tau_{j}\right) \phi\right\| .
$$

Since $A^{z}\left(\tau_{j}\right) \phi \rightarrow A\left(\tau_{j}\right) \phi, z \downarrow 0, \exists z_{0}$ such that

$$
0<z \leqslant z_{0} \Rightarrow\left\|A^{z}\left(\tau_{j}\right) \phi-A\left(\tau_{j}\right) \phi\right\|<\varepsilon / 6 t .
$$

Hence, if $0<z_{1}, z_{2} \leqslant z_{0}$ then

$$
\left\|A^{z_{1}}\left(\tau_{j}\right) \phi-A^{z_{2}}\left(\tau_{j}\right) \phi\right\| \leqslant\left\|A\left(\tau_{j}\right) \phi-A^{z_{1}}\left(\tau_{j}\right) \phi\right\|+\left\|A\left(\tau_{j}\right) \phi-A^{z_{2}}\left(\tau_{j}\right) \phi\right\| \leqslant \varepsilon / 3 t,
$$

so that

$$
\left\|Q_{\lambda}^{z_{1}}[t, 0] \phi-Q_{\lambda}^{z_{2}}[t, 0] \phi\right\| \leqslant e^{-\lambda T} \sum_{n=0}^{\infty} \frac{(\lambda T)^{n}}{n !} \sum_{j=1}^{n} \Delta t_{j}\left(\frac{\varepsilon}{3 t}\right) \leqslant \frac{\varepsilon}{3} .
$$


Combining this with (A) and (B), we see that for $z_{1}, z_{2} \leqslant z_{0}$,

$$
\left\|Q^{z_{1}}[t, 0] \phi-Q^{z_{2}}[t, 0] \phi\right\|<\varepsilon
$$

so that $\lim _{z \downarrow 0} Q^{z}[t, 0] \phi=Q[t, 0] \phi$ exists. It is easy to see that $Q[t, 0] \phi=Q[t, s] \phi+$ $Q[s, 0] \phi$. Q.E.D.

Let us set

$$
Q_{\lambda}[t, 0]=e^{-\lambda T} \sum_{n=0}^{\infty} \frac{(\lambda T)^{n}}{n !}\left(\sum_{j=1}^{n} \Delta t_{j} A\left(\tau_{j}\right)\right)
$$

since $A^{z}\left(\tau_{j}\right) \stackrel{s}{\rightarrow} A\left(\tau_{j}\right)$, it is easy to see that

$$
\lim _{z \downarrow 0} Q_{\lambda}^{z}[t, 0] \phi=Q_{\lambda}[t, 0] \phi, \quad \forall \phi \in D .
$$

THEOREM 1.2. (1) $\lim _{\lambda \rightarrow \infty} Q_{\lambda}[t, 0] \phi=Q[t, 0] \phi$, that is (from Theorem 1.1)

$$
\lim _{\lambda \rightarrow \infty} \lim _{z \downarrow 0} Q_{\lambda}^{z}[t, 0] \phi=\lim _{z \downarrow 0} \lim _{\lambda \rightarrow \infty} Q_{\lambda}^{z}[t, 0] \phi=Q[t, 0] \phi .
$$

(2) $Q[t, 0]$ has a closure which is the generator of a strongly continuous contraction semigroup on $\otimes_{\tau}^{\alpha} B(\tau)$.

Proof of (1). Use the above remarks and Theorem 1.1 to see that since

$$
\begin{aligned}
\| Q_{\lambda}[t, 0] \phi & -Q[t, 0] \phi\|\leqslant\| Q_{\lambda}^{z}[t, 0] \phi-Q_{\lambda}[t, 0] \phi \| \\
& +\left\|Q_{\lambda}^{z}[t, 0] \phi-Q^{z}[t, 0] \phi\right\|+\left\|Q^{z}[t, 0] \phi-Q[t, 0] \phi\right\|,
\end{aligned}
$$

we may choose $z$ so that the first and third terms are small, then choose $\lambda$ so that the second term is small, hence $\lim _{\lambda \rightarrow \infty} Q_{\lambda}[t, 0] \phi=Q[t, 0] \phi$.

Proof of (2). Let $\phi \in D$ and $J_{\phi}$ be a duality map for $\phi$. We know that $Q_{n}[t, 0]$ is a generator for each $n$ so that $\operatorname{Re}\left\langle Q_{n}[t, 0] \phi, J_{\phi}\right\rangle \leqslant 0 \forall n$ (i.e., $Q_{n}[t, 0]$ is dissipative). Now

$$
\operatorname{Re}\left\langle Q_{\lambda}[t, 0] \phi, J_{\phi}\right\rangle=\lim _{N \rightarrow \infty} e^{-\lambda T} \sum_{n=0}^{N} \frac{(\lambda T)^{n}}{n !} \operatorname{Re}\left\langle Q_{n}[t, 0] \phi, J_{\phi}\right\rangle .
$$

Hence $\operatorname{Re}\left\langle Q_{\lambda}[t, 0] \phi, J_{\phi}\right\rangle \leqslant 0$ so that $Q_{\lambda}[t, 0]$, being densely defined and dissipative, has a closure which is the unique generator of a contraction semigroup on $\otimes_{\tau}^{\alpha} B(\tau)$. Since $\lim _{\lambda \rightarrow \infty} Q_{\lambda}[t, 0] \phi=Q[t, 0] \phi$, we have

$$
\operatorname{Re}\left\langle Q[t, 0] \phi, J_{\phi}\right\rangle=\operatorname{Re}\left\langle Q_{\lambda}[t, 0] \phi, J_{\phi}\right\rangle+\operatorname{Re}\left\langle\left(Q[t, 0] \phi-Q_{\lambda}[t, 0] \phi\right), J_{\phi}\right\rangle .
$$

As the first term is $\leqslant 0$ we get

$$
\operatorname{Re}\left\langle Q[t, 0] \phi, J_{\phi}\right\rangle \leqslant \operatorname{Re}\left\langle\left(Q[t, 0] \phi-Q_{\lambda}[t, 0] \phi\right), J_{\phi}\right\rangle .
$$

Letting $\lambda \rightarrow \infty$, we get $\operatorname{Re}\left\langle Q[t, 0] \phi, J_{\phi}\right\rangle \leqslant 0$ so $Q[t, 0]$ has a closure which is dissipative and densely defined. We complete our proof by showing that the range of $(\lambda-Q[t, 0])$ is $\otimes_{\tau}^{\alpha} B(\tau)$ for $\lambda>0$. The proof of this part is identical to that of [G1, Theorem 4.3] (see also Lemma 4.2). Q.E.D.

Definition 1.1 . We call $Q[t, 0]$ the time-ordered integral of the family $\{A(\tau) \mid \tau \in$ $[0, t]\}$ and write it as

$$
Q[t, 0]=\int_{0}^{t} A(\tau) d \tau
$$


We also denote $Q^{z}[t, 0]$ by

$$
Q^{z}[t, 0]=\int_{0}^{t} A^{z}(\tau) d \tau
$$

The notation is partially justified by

THEOREM 1.3. $\varepsilon^{-1} Q^{z}[t+\varepsilon, t] \stackrel{c}{\rightarrow} A^{z}(t)$ as $\varepsilon \downarrow 0$ in the sense that

$$
\lim _{\varepsilon \downarrow 0} \frac{1}{\varepsilon}\left\|Q^{z}[t+\varepsilon, t]-\int_{t}^{t+\varepsilon} E[\tau, t] A^{z}(t) d \tau\right\|=0 .
$$

Proof. Since

$$
\lim _{\lambda \rightarrow \infty} \sum_{n=0}^{\infty} e^{-\lambda T} \frac{(\lambda T)^{n}}{n !} \sum_{j=1}^{n} E\left[\tau_{j}, t\right] A^{z}(t) \Delta t_{j}=\int_{t}^{t+\varepsilon} d \tau E[\tau, t] A^{z}(t),
$$

then

$$
\begin{gathered}
\left\|Q_{\lambda}^{z}[t+\varepsilon, t]-\sum_{n=0}^{\infty} e^{-\lambda T} \frac{(\lambda T)^{n}}{n !} \sum_{j=1}^{n} \Delta t_{j} E\left[\tau_{j}, t\right] A^{z}(t)\right\| \\
\leqslant \sum_{n=0} e^{-\lambda T} \frac{(\lambda T)^{n}}{n !} \sum_{j=1}^{n} \Delta t_{j}\left\|A^{z}\left(\tau_{j}\right)-E\left[\tau_{j}, t\right] A^{z}(t)\right\| .
\end{gathered}
$$

Since $\left\|A^{z}\left(\tau_{j}\right)-E\left[\tau_{j}, t\right] A^{z}(t)\right\|=\left\|\tilde{A}^{z}\left(\tau_{j}\right)-\tilde{A}^{z}(t)\right\|$, where the norm on the left is in $L\left[\otimes_{\tau}^{\alpha} B(\tau)\right]$ and that on the right in $L[B]$ (recall that $L[B]$ is the space of bounded operators on $B$ ), we have

$$
\begin{aligned}
\| Q^{z}[t+\varepsilon, t]- & \int_{t}^{t+\varepsilon} E[\tau, t] A^{z}(t) d \tau \| \\
& =\lim _{\lambda \rightarrow \infty}\left\|Q_{\lambda}^{z}[t+\varepsilon, t]-\sum_{n=0}^{\infty} e^{-\lambda T} \frac{(\lambda T)^{n}}{n !} \sum_{j=1}^{n} \Delta t_{j} E\left[\tau_{j}, t\right] A^{z}(t)\right\| \\
& \leqslant \lim _{\lambda \rightarrow \infty} \sum_{n=0}^{\infty} e^{-\lambda T} \frac{(\lambda T)^{n}}{n !} \sum_{j=1}^{n} \Delta t_{j}\left\|\tilde{A}^{z}\left(\tau_{j}\right)-\tilde{A}^{z}(t)\right\| \\
& =\int_{t}^{t+\varepsilon_{\|}}\left\|\tilde{A}^{z}(\tau)-\tilde{A}^{z}(t)\right\| d \tau .
\end{aligned}
$$

Hence

$$
\begin{aligned}
\frac{1}{\varepsilon}\left\|Q^{z}[t+\varepsilon, t]-\int_{t}^{t+\varepsilon} E[\tau, t] A^{z}(\tau) d \tau\right\| & \\
& \leqslant \frac{1}{\varepsilon} \int_{t}^{t+\varepsilon}\left\|\tilde{A}^{z}(\tau)-\tilde{A}^{z}(t)\right\| d \tau \rightarrow 0, \quad \varepsilon \downarrow 0 . \quad \text { Q.E.D }
\end{aligned}
$$

COROLlaRY 1.1. $\forall \phi \in D, \varepsilon^{-1} Q[t+\varepsilon, t] \phi \stackrel{c}{\rightarrow} A(t) \phi$.

We now define $U^{z}[t, 0]$ and $U[t, 0]$ by

$$
U^{z}[t, 0]=\exp \left\{Q^{z}[t, 0]\right\}, \quad U[t, 0]=\exp \{Q[t, 0]\} .
$$


THEOREM 1.4.

$$
\begin{gathered}
U^{z}[t, 0]=U^{z}[t, s] U^{z}[s, 0]=U^{z}[s, 0] U^{z}[t, s], \quad 0 \leqslant s<t . \\
s-\lim _{z \downarrow 0} U^{z}[t, 0]=U[t, 0]
\end{gathered}
$$

and

(3)

$$
\begin{gathered}
U[t, 0]=U[t, s] U[s, 0]=U[s, 0] U[t, s] . \\
\frac{\partial U^{z}[t, 0]}{\partial t}=A^{z}(t) U^{z}[t, 0], \quad \frac{\partial U[t, 0]}{\partial t}=A(t) U[t, 0],
\end{gathered}
$$

where (1.5) is defined in the strong chronological sense.

Proof. (1) and (2) follow from Theorem 1.1. To prove (3) note that $Q^{z}[t, t]=0$ and

$$
U^{z}[t+\varepsilon, t]=\sum_{k=0}^{\infty} \frac{Q^{z}[t+\varepsilon, t]^{k}}{k !}
$$

since

$$
\begin{aligned}
\frac{U^{z}[t+\varepsilon, 0]}{\varepsilon}-U^{z}[t, 0] & =\frac{U^{z}[t+\varepsilon, t]-I}{\varepsilon} U^{z}[t, 0] \\
& =\frac{1}{\varepsilon}\left[\sum_{k=0}^{\infty} \frac{Q^{z}[t+\varepsilon, t]^{k}}{k !}-I\right] U^{z}[t, 0] \\
& =\left[\frac{Q^{z}[t+\varepsilon, t]}{\varepsilon}+\frac{Q^{z}[t+\varepsilon, t]}{\varepsilon} \sum_{k=2}^{\infty} \frac{Q^{z}[t+\varepsilon, t]^{k-1}}{k !}\right] U^{z}[t, 0] \\
& \rightarrow A^{z}(t) U^{z}[t, 0]
\end{aligned}
$$

by Theorem 1.3, we have

$$
\frac{\partial U^{z}[t, 0]}{\partial t}=A^{z}(t) U^{z}[t, 0]
$$

Taking strong limits on both sides gives

$$
\frac{\partial U[t, 0]}{\partial t}=A(t) U[t, 0] \text {. Q.E.D. }
$$

Let us close this section by noting that $U[t, 0]$ can also be obtained as the strong limit of $\exp \left\{Q_{\lambda}[t, 0]\right\}$. Since this result will be used later, and also because it has some independent interest, we prove 
LEMMA 1.1.

$$
U[t, 0]=\underset{\lambda \rightarrow \infty}{s-\lim } \exp \left\{Q_{\lambda}[t, 0]\right\}
$$

Proof.

$$
\begin{aligned}
\left\|U[t, 0] \phi-\exp \left\{Q_{\lambda}[t, 0]\right\} \phi\right\| \\
\quad=\left\|\int_{0}^{1}\left[\frac{d}{d v} \exp \{(1-v) Q[t, 0]\} \exp \left\{v Q_{\lambda}[t, 0]\right\} \phi\right] d v\right\| \\
\quad=\left\|\int_{0}^{1} d v\left[\exp \{(1-v) Q[t, 0]\} \exp \left\{v Q_{\lambda}[t, 0]\right\}\right]\left[Q[t, 0] \phi-Q_{\lambda}[t, 0] \phi\right]\right\| \\
\quad \leqslant \int_{0}^{1} d v\left\|Q[t, 0] \phi-Q_{\lambda}[t, 0] \phi\right\|=\left\|Q[t, 0] \phi-Q_{\lambda}[t, 0] \phi\right\| .
\end{aligned}
$$

This last term approaches zero as $\lambda \rightarrow \infty$ by Theorem 1.2. Q.E.D.

2. As is generally the case, the desire for a nice method and approach has the effect of submerging motivation, thus making simple ideas appear abstract and uninviting. The previous section provides us with almost all the tools required for a general study of linear evolution equations. Already we can resolve a number of fundamental issues such as:

(1) conditions under which the sum of two generators is a generator; and

(2) conditions under which the perturbation expansion is mathematically justified.

We will provide quite satisfactory answers to these questions in §3; however, there are two disadvantages inherent in the approach used in $\$ 1$ (other than a lack of motivation for the method). The first, and most severe, is the fact that the present approach hides the natural connection between time-ordered operators and path integrals (of the "sum over histories type") as envisioned by Feynman [F1].

The second disadvantage is the fact that we can only approximate $U[t, 0]$ in the strong sense. As will be seen in subsequent work, this can create certain complications in relating our approach to conventional attempts.

Let us define $U_{n}[t, 0], U_{n}^{z}[t, 0], \bar{U}_{n}[t, 0], \bar{U}_{n}^{z}[t, 0]$ by

$$
\begin{aligned}
& \left\{\begin{array}{l}
U_{n}[t, 0]=\exp \left\{\sum_{j=1}^{n} \Delta t_{j} A\left(\tau_{j}\right)\right\}, \\
U_{n}^{z}[t, 0]=\exp \left\{\sum_{j=1}^{n} \Delta t_{j} A^{z}\left(\tau_{j}\right)\right\},
\end{array}\right. \\
& \left\{\begin{array}{l}
\bar{U}_{n}[t, 0]=\exp \left\{\sum_{j=1}^{n} \int_{t_{j-1}}^{t_{j}} E\left[\tau_{j}, \tau\right] A(\tau) d \tau\right\}, \\
\bar{U}_{n}^{z}[t, 0]=\exp \left\{\sum_{j=1}^{n} \int_{t_{j-1}}^{t_{j}} E\left[\tau_{j}, \tau\right] A^{z}(\tau) d \tau\right\} .
\end{array}\right.
\end{aligned}
$$


With the above expressions, define $U_{\lambda}[t, 0], \bar{U}_{\lambda}[t, 0]$ by

$$
\left\{\begin{array}{l}
U_{\lambda}[t, 0]=e^{-\lambda T} \sum_{n=0}^{\infty} \frac{(\lambda T)^{n}}{n !} U_{n}[t, 0], \\
\bar{U}_{\lambda}[t, 0]=e^{-\lambda T} \sum_{n=0}^{\infty} \frac{(\lambda T)^{n}}{n !} \bar{U}_{n}[t, 0] .
\end{array}\right.
$$

Similar definitions give $U_{\lambda}^{z}[t, 0]$ and $\bar{U}_{\lambda}^{z}[t, 0]$.

THEOREM 2.1. (1) $\left\|U_{\lambda}[t, 0]\right\|,\left\|\bar{U}_{\lambda}[t, 0]\right\| \leqslant 1$.

(2) $\lim _{\lambda \rightarrow \infty} U_{\lambda}[t, 0]=U[t, 0]$ and $\lim _{\lambda \rightarrow \infty} \bar{U}_{\lambda}[t, 0]=\bar{U}[t, 0]$ exist. Both limits are in the uniform operator topology.

Proof. (1)

$$
\left\|U_{\lambda}[t, 0]\right\| \leqslant \sum_{n=0}^{\infty} e^{-\lambda T} \frac{(\lambda T)^{n}}{n !}\left\|U_{n}[t, 0]\right\| \leqslant 1
$$

(2)

$$
\left\|U_{\lambda_{1}}[t, 0]-U_{\lambda_{2}}[t, 0]\right\| \leqslant \sum_{n=0}^{\infty}\left|\lambda_{1}^{n} e^{-\lambda_{1} T}-\lambda_{2}^{n} e^{-\lambda_{2} T}\right| \frac{T^{n}}{n !} .
$$

Since each term in the series $\rightarrow 0$ as $\lambda_{1}, \lambda_{2} \rightarrow \infty$, we see that $\left\{U_{\lambda}[t, 0] \mid \lambda>0\right\}$ is a Cauchy family in the uniform operator topology, so that $\lim _{\lambda \rightarrow \infty} U_{\lambda}[t, 0]=U[t, 0]$ exists. It is straightforward to prove the same results for $\bar{U}_{\lambda}[t, 0]$. Q.E.D.

THEOREM 2.2. $U[t, 0]=\bar{U}[t, 0]$.

Proof. It is clear from $\S 1$ that

$$
s-\lim _{z \downarrow 0} U_{n}^{z}[t, 0]=U_{n}[t, 0] \text { and } s-\lim _{z \downarrow 0} \bar{U}_{n}^{z}[t, 0]=\bar{U}_{n}[t, 0],
$$

so it suffices to prove $U^{z}[t, 0]=\bar{U}^{z}[t, 0]$.

$$
\left\|\bar{U}_{n}^{z}[t, 0]-U_{n}^{z}[t, 0]\right\|=\left\|\prod_{j=1}^{n} \exp \left\{\int_{t_{j-1}}^{t_{j}} E\left[\tau_{j}, \tau\right] A^{z}(\tau) d \tau\right\}-\prod_{j=1}^{n} \exp \left\{\Delta t_{j} A^{z}\left(\tau_{j}\right)\right\}\right\| .
$$

We now use the fact that

$$
\left|\prod_{j=1}^{n} e^{x_{j}}-\prod_{j=1}^{n} e^{y_{j}}\right|=\left|\sum_{j=1}^{n} \prod_{i=1}^{j-1} e^{x_{i}}\left(e^{x_{j}}-e^{y_{j}}\right) \prod_{i=j+1}^{n} e^{y_{j}}\right|
$$

(in our case $\left|e^{x_{i}}\right|,\left|e^{y_{i}}\right| \leqslant 1$ ) to obtain

$$
\left\|U_{n}^{z}[t, 0]-\bar{U}_{n}^{z}[t, 0]\right\| \leqslant \sum_{j=1}^{n}\left\|\exp \left\{\int_{t_{j-1}}^{t_{j}} E\left[\tau_{j}, \tau\right] A^{z}\left(\tau_{j}\right) d \tau\right\}-\exp \left\{\Delta t_{j} A^{z}\left(\tau_{j}\right)\right\}\right\| .
$$


Let us consider the $j$ th term in (2.4):

(2.5)

$$
\begin{aligned}
& \left\|\exp \left\{\int_{t_{j-1}}^{t_{j}} E\left[\tau_{j}, \tau\right] A^{z}(\tau) d \tau\right\}-\exp \left\{\Delta t_{j} A^{z}\left(\tau_{j}\right)\right\}\right\| \\
& =\left\|\int_{0}^{1} d v\left(\frac{d}{d v} \exp \left\{v \int_{t_{j-1}}^{t_{j}} E\left[\tau_{j}, \tau\right] A^{z}(\tau) d \tau\right\} \exp \left\{(1-v) \Delta t_{j} A^{z}\left(\tau_{j}\right)\right\}\right)\right\| \\
& \leqslant \int_{0}^{1} d v \| \exp \left\{v \int_{t_{j-1}}^{t_{j}} E\left[\tau_{j}, \tau\right] A^{z}(\tau) d \tau\right\} \\
& \quad \times\left\{\int_{t_{j-1}}^{t_{j}} E\left[\tau_{j}, \tau\right] A^{z}(\tau) d \tau-\Delta t_{j} A^{z}\left(\tau_{j}\right) \exp \left\{(1-v) \Delta t_{j} A^{z}\left(\tau_{j}\right)\right\}\right\} \| \\
& \leqslant\left\|\int_{t_{j-1}}^{t_{j}}\left(E\left[\tau_{j}, \tau\right] A^{z}(\tau)-A^{z}\left(\tau_{j}\right)\right) d \tau\right\| \\
& \leqslant \int_{t_{j-1}}^{t_{j}}\left\|E\left[\tau_{j}, \tau\right] A^{z}(\tau)-A^{z}\left(\tau_{j}\right)\right\| d \tau .
\end{aligned}
$$

As in $§ 1$,

$$
\left\|E\left[\tau_{j}, \tau\right] A^{z}(\tau)-A^{z}\left(\tau_{j}\right)\right\|=\left\|\tilde{A}^{z}(\tau)-\tilde{A}^{z}\left(\tau_{j}\right)\right\|,
$$

so that using (2.6) and (2.5) in (2.4), we have

$$
\left\|U_{n}^{z}[t, 0]-\bar{U}_{n}^{z}[t, 0]\right\| \leqslant \sum_{j=1}^{n} \int_{t_{j-1}}^{t_{j}}\left\|\tilde{A}^{z}(\tau)-\tilde{A}^{z}\left(\tau_{j}\right)\right\| d \tau .
$$

Choose $N$ such that

$$
n \geqslant N \Rightarrow\left\|A^{z}(\tau)-A^{z}\left(\tau_{j}\right)\right\|<\frac{\varepsilon(z)}{t},
$$

which can be done since $\tilde{A}^{z}(t)$ is continuous; this means that for $n \geqslant N$,

$$
\left\|U_{n}^{z}[t, 0]-\bar{U}_{n}^{z}[t, 0]\right\| \leqslant \sum_{j=1}^{n} \int_{t_{n-1}}^{t_{j}} d \tau\left(\frac{\varepsilon(z)}{t}\right) \leqslant \varepsilon(z) .
$$

Now

$$
\begin{aligned}
\left\|U_{\lambda}^{z}[t, 0]-\bar{U}_{\lambda}^{z}[t, 0]\right\| \leqslant & e^{-\lambda T} \sum_{n=0}^{\infty} \frac{(\lambda T)^{n}}{n !}\left\|U_{n}^{z}[t, 0]-\bar{U}_{n}^{z}[t, 0]\right\| \\
= & e^{-\lambda T} \sum_{n=0}^{N} \frac{(\lambda T)^{n}}{n !}\left\|U_{n}^{z}[t, 0]-\bar{U}_{n}^{z}[t, 0]\right\| \\
& +e^{-\lambda T} \sum_{n=N+1}^{\infty} \frac{(\lambda T)^{n}}{n !}\left\|U_{n}^{z}[t, 0]-\bar{U}_{n}^{z}[t, 0]\right\| .
\end{aligned}
$$


Use (2.8) in the second part of (2.9) to get

$$
\left\|U_{\lambda}^{z}[t, 0]-\bar{U}_{\lambda}^{z}[t, 0]\right\| \leqslant e^{-\lambda T} \sum_{n=0}^{N} \frac{(\lambda T)^{n}}{n !}\left\|U_{n}^{z}[t, 0]-\bar{U}_{n}^{z}[t, 0]\right\|+\varepsilon(z) .
$$

Letting $\lambda \rightarrow \infty$ we get

$$
\left\|U^{z}[t, 0]-\bar{U}^{z}[t, 0]\right\| \leqslant \varepsilon(z) .
$$

Since $\varepsilon(z)$ is arbitrary we see that

$$
U^{z}[t, 0]=\bar{U}^{z}[t, 0] \quad \forall z>0 .
$$

Hence $U[t, 0]=\bar{U}[t, 0]$. Q.E.D.

It is intuitively clear that the above $U[t, 0]$ and that defined in (1.4) are the same. However, a direct proof requires some additional work.

Let us set $\Delta t_{n}=t-t_{n-1}$ in our definition of $U_{n}[t, 0]$, for each $n$.

LEMMA 2.1. Suppose $\tau_{n}=\tau_{n}(\lambda)$ satisfies $\lim _{\lambda \rightarrow \infty} \tau_{n}=\lim _{n \rightarrow \infty} \tau_{n}=t$. Then $\sum_{n=0}^{\infty} e^{-\lambda T}\left((\lambda T)^{n} / n !\right) A\left(\tau_{n}\right)$ converges strongly in the chronological sense to $A(t)$; that is

$$
\lim _{\lambda \rightarrow \infty} \sum_{n=0}^{\infty} e^{-\lambda T} \frac{(\lambda T)^{n}}{n !}\left\|A\left(\tau_{n}\right) \phi-E\left[\tau_{n}, t\right] A(t) \phi\right\|=0 \quad \text { for } \phi \in D .
$$

Proof. It suffices to prove the result for $A^{z}(t)$. Now

$$
\left\|E\left[\tau_{n}, t\right] A^{z}(t)-A^{z}\left(\tau_{n}\right)\right\|=\left\|\tilde{A}^{z}(t)-\tilde{A}^{z}\left(\tau_{n}\right)\right\| .
$$

Since $\tilde{A}^{z}(\tau)$ is continuous, given $\varepsilon=\varepsilon(z), \exists N$ such that $n \geqslant N \Rightarrow\left\|\tilde{A}^{z}(t)-\tilde{A}^{z}\left(\tau_{n}\right)\right\|<$ $\varepsilon(z)$. Thus

$$
\begin{aligned}
& \sum_{n=0}^{\infty} e^{-\lambda T} \frac{(\lambda T)^{n}}{n !}\left\|A^{z}\left(\tau_{n}\right)-E\left[\tau_{n}, t\right] A^{z}(t)\right\| \\
& \leqslant \sum_{n=0}^{N} e^{-\lambda T} \frac{(\lambda T)^{n}}{n !}\left\|A^{z}\left(\tau_{n}\right)-E\left[\tau_{n}, t\right] A^{z}(t)\right\|+\varepsilon
\end{aligned}
$$

Letting $\lambda \rightarrow \infty$ we get

$$
\lim _{\lambda \rightarrow \infty} \sum_{n=0}^{\infty} e^{-\lambda T} \frac{(\lambda T)^{n}}{n !}\left\|A^{z}\left(\tau_{n}\right)-E\left[\tau_{n}, t\right] A^{z}(t)\right\| \leqslant \varepsilon . \quad \text { Q.E.D. }
$$

TheOREM 2.3. (1)

$$
\frac{\partial U_{\lambda}^{z}[t, 0]}{\partial t}=\sum_{n=0}^{\infty} e^{-\lambda T} \frac{(\lambda T)^{n}}{n !} A^{z}\left(\tau_{n}\right) U_{n}^{z}[t, 0]
$$

and

$$
\frac{\partial U^{z}[t, 0]}{\partial t}=A^{z}(t) U^{z}[t, 0] .
$$

(2)

$$
\frac{\partial U_{\lambda}[t, 0]}{\partial t}=\sum_{n=0}^{\infty} e^{-\lambda T} \frac{(\lambda T)^{n}}{n !} A\left(\tau_{n}\right) U_{n}[t, 0]
$$


and

$$
\frac{\partial U[t, 0]}{\partial t}=A(t) U[t, 0] .
$$

In (2.11) the derivative is in the chronological sense and in (2.12) it is in the strong chronological sense.

Proof of (1). Let $N$ be arbitrary and define $V_{\lambda}^{N}[z,[t, 0]]$ by

$$
V_{\lambda}^{N}[z,[t, 0]]=\sum_{n=0}^{N} e^{-\lambda T} \frac{(\lambda T)^{n}}{n !} U_{n}^{z}[t, 0] .
$$

Since $\Delta t_{n}=t-t_{n-1}$, it follows that

$$
\frac{\partial V_{\lambda}^{N}}{\partial t}=e^{-\lambda T} \sum_{n=0}^{N} \frac{(\lambda T)^{n}}{n !} A^{z}\left(\tau_{n}\right) U_{n}^{z}[t, 0]
$$

and

$$
\begin{aligned}
& \left\|\frac{\partial V_{\lambda}^{N_{1}}}{\partial t}-\frac{\partial V_{\lambda}^{N_{2}}}{\partial t}\right\| \leqslant \sum_{n=N_{1}+1}^{N_{2}} e^{-\lambda T} \frac{(\lambda T)^{n}}{n !}\left\|A^{z}\left(\tau_{n}\right) U_{n}^{z}[t, 0]\right\| \\
& \leqslant\left(N_{2}-N_{1}-1\right)\left(\frac{2}{z}\right) e^{2 t / z-\lambda T} \sup _{N_{1}+1 \leqslant n \leqslant N_{2}} \frac{(\lambda T)^{n}}{n !} .
\end{aligned}
$$

This last term can be made small if $N_{1}$ and $N_{2}$ are large, so $\partial U_{\lambda}^{z} / \partial t=$ $\lim _{N \rightarrow \infty}\left(\partial V_{\lambda}^{N} / \partial t\right)$ exists with bound

$$
\left\|\frac{\partial U_{\lambda}^{z}}{\partial t}\right\| \leqslant\left(\frac{2}{z}\right) e^{2 t / z}, \quad \frac{\partial U_{\lambda}^{z}}{\partial t}=e^{-\lambda T} \sum_{n=0}^{\infty} \frac{(\lambda T)^{n}}{n !} A^{z}\left(\tau_{n}\right) U_{n}^{z}[t, 0] .
$$

To see that $\partial U_{\lambda}^{z} / \partial t c$-converges to $A^{z}(t) U^{z}[t, 0]$, first note that the limit exists with bound $(2 / z) e^{2 t / z}$.

$$
\begin{aligned}
\| \frac{\partial U_{\lambda}^{z}}{\partial t}-e^{-\lambda T} \sum_{n=0}^{\infty} & \frac{(\lambda T)^{n}}{n !} E\left[\tau_{n}, t\right] A^{z}(t) U^{z}[t, 0] \| \\
= & e^{-\lambda t}\left\|\sum_{n=0}^{\infty} \frac{(\lambda T)^{n}}{n !}\left\{A^{z}\left(\tau_{n}\right) U_{n}^{z}[t, 0]-E\left[\tau_{n}, t\right] A^{z}(t) U^{z}[t, 0]\right\}\right\| \\
\leqslant & \sum_{n=0}^{\infty} e^{-\lambda T} \frac{(\lambda T)^{n}}{n !}\left\|A^{z}\left(\tau_{n}\right) U_{n}^{z}[t, 0]-E\left[\tau_{n}, t\right] A^{z}(t) U_{n}^{z}[t, 0]\right\| \\
& +\sum_{n=0}^{\infty} e^{-\lambda T} \frac{(\lambda T)^{n}}{n !}\left\|\left(E\left[\tau_{n}, t\right] A^{z}(t)\right)\left(U_{n}^{z}[t, 0]-U^{z}[t, 0]\right)\right\| \\
\leqslant & e^{2 t / z-\lambda T} \sum_{n=0}^{\infty} \frac{(\lambda T)^{n}}{n !}\left\|A^{z}\left(\tau_{n}\right)-E\left[\tau_{n}, t\right] A^{z}(t)\right\| \\
& +\frac{2}{z} e^{-\lambda T} \sum_{n=0}^{\infty} \frac{(\lambda T)^{n}}{n !}\left\|U_{n}^{z}[t, 0]-U^{z}[t, 0]\right\| .
\end{aligned}
$$


The first term $\rightarrow 0$ by Lemma 2.1 , the second by definition of $U^{z}[t, 0]$, so that

$$
\frac{\partial U^{z}}{\partial t}=\lim _{\lambda \rightarrow \infty} \frac{\partial U_{\lambda}^{z}}{\partial t}=A^{z}(t) U^{z}[t, 0]
$$

in the chronological sense. The proof of (2) now follows from strong limits. Q.E.D.

We may now use the uniqueness of the initial value problem (in the strong sense) to conclude that the evolution operator obtained in this section is the same as that of $\S 1$.

3. In [G1] it was shown that the time-ordered sum of two generators of contraction semigroups is a generator almost everywhere in the weak operator topology. The result depended on the assumed reflexive nature of the underlying space. The fact of the matter is that under the most natural conditions the tensor product of two reflexive Banach spaces need not be reflexive. This result goes back to Shatten [5], the father of the subject. (See also Grothendieck [Gr].) Later work by Holub $[\mathbf{H}]$ provides conditions on two Banach spaces if the crossnorm is either $\lambda$ (least) or $\gamma$ (greatest). Holub also provides other interesting results.

If $X$ is a reflexive infinite-dimensional Banach space, for example, then both $X \otimes^{\lambda} X^{*}$ and $X \otimes{ }^{\gamma} X^{*}$ are not reflexive. A special case of this general result was known by Shatten and Grothendieck. Except for the small contribution of Gill [G2], very little is known about infinite tensor products of Banach spaces. This means that the theorem proved in [G1] can be depended on only when all the underlying spaces are Hilbert with the unique Hilbert space crossnorm completion of von Neumann. In this section we remove the space restrictions and strengthen the result from the weak to the uniform operator topology.

Let us suppose we have two families of time-ordered operators $\left\{A_{1}(\tau) \mid \tau \in[0, T]\right\}$ and $\left\{A_{2}(\tau) \mid \tau \in[0, T]\right\}$ with domains $D_{1}(\tau)$ and $D_{2}(\tau)$ for each $\tau$. We assume that both families are strong $c$-continuous generators of contraction semigroups. If $P_{n}$ is a standard partition of $[0, t]$, let $\tau_{j}, s_{j} \in\left[t_{j-1}, t_{j}\right]$ and define

$$
\left\{\begin{array}{l}
U_{n}^{0}[t, 0]=\exp \left\{\sum_{j=1}^{n} \Delta t_{j}\left[A_{1}\left(\tau_{j}\right)+A_{2}\left(s_{j}\right)\right]\right\}, \\
U_{n}^{1}[t, 0]=\exp \left\{\sum_{j=1}^{n} \Delta t_{j} A_{1}\left(\tau_{j}\right)\right\}, \\
U_{n}^{2}[t, 0]=\exp \left\{\sum_{n=1}^{n} \Delta t_{j} A_{2}\left(s_{j}\right)\right\} .
\end{array}\right.
$$

Since we have not assumed any relationship between $D_{1}(\tau)$ and $D_{2}(s), U_{n}^{0}[t, 0]$ is well defined except when $\tau_{j}=s_{j}$ for some $j$, otherwise $U_{n}^{p}[t, 0]=U_{n}^{1}[t, 0] U_{n}^{2}[t, 0]=$ $U_{n}^{2}[t, 0] U_{n}^{1}[t, 0]$. Define $U_{\lambda}^{i}[t, 0], i=0,1,2$, by

$$
U_{\lambda}^{i}[t, 0]=\exp (-\lambda T) \sum_{n=0}^{\infty} \frac{(\lambda T)^{n}}{n !} U_{n}^{i}[t, 0] .
$$

THEOREM 3.1. (1) $\lim _{\lambda \rightarrow \infty} U_{\lambda}^{0}[t, 0]=U^{0}[t, 0]$ exists a.e.

(2) $U^{0}[t, 0]=U^{1}[t, 0] U^{2}[t, 0]=U^{2}[t, 0] U^{1}[t, 0]$ a.e. 
Proof. (1) is clear; to prove (2) let $I=I_{1} \cup I_{2}: I_{1}, I_{2}$ dense in $[0, T] ; I_{1} \cap I_{2}$ empty. Choose $\tau_{j} \in I_{1}, s_{j} \in I_{2}$ so that $U_{n}^{0}[t, 0]$ and $U_{\lambda}^{0}[t, 0]$ are well defined. Then

$$
\begin{aligned}
U_{\lambda}^{1}[t, 0] U_{\lambda}^{2}[t, 0] & =\exp (-2 \lambda T)\left[\sum_{m=0}^{\infty} \frac{(\lambda T)^{m}}{m !} U_{m}^{1}[t, 0]\right]\left[\sum_{l=0}^{\infty} \frac{(\lambda T)^{l}}{l !} U_{l}^{2}[t, 0]\right] \\
& =\sum_{n=0}^{\infty} e^{-2 \lambda T} \sum_{k=0}^{n}\left[\frac{(\lambda T)^{k}}{k !} U_{k}^{1}[t, 0]\right]\left[\frac{(\lambda T)^{n-k}}{(n-k) !} U_{n-k}^{2}[t, 0]\right] \\
& =\sum_{n=0}^{\infty} e^{-2 \lambda T} \frac{(\lambda T)^{n}}{n !} \sum_{k=0}^{n}\left[\begin{array}{l}
n \\
k
\end{array}\right] U_{k}^{1}[t, 0] U_{n-k}^{2}[t, 0] .
\end{aligned}
$$

Set $\tilde{P}_{n}=P_{k} \cup P_{n-k}$, where we count points in $P_{k}$ and $P_{n-k}$ as distinct (which is required since the $\tau_{j}$ and $s_{j}$ are distinct). We now have

$$
U_{\lambda}^{1}[t, 0] U_{\lambda}^{2}[t, 0]=e^{-2 \lambda T} \sum_{n=0}^{\infty} \frac{(2 \lambda T)^{n}}{n !} \tilde{U}_{n}^{0}[t, 0] .
$$

The " " " is used to denote the use of a different family of partitions $\tilde{P}_{n}$; however, it is still true that $\lim _{n \rightarrow \infty}\left|\tilde{P}_{n}\right|=0$. Comparing (3.3) with (3.2) we see that

$$
U_{\lambda}^{1}[t, 0] U_{\lambda}^{2}[t, 0]=\tilde{U}_{2 \lambda}^{0}[t, 0] .
$$

The same calculation gives

$$
U_{\lambda}^{2}[t, 0] U_{\lambda}^{1}[t, 0]=\tilde{U}_{2 \lambda}^{0}[t, 0] .
$$

It should be observed that the same $\tau_{j}$ and $s_{j}$ appear on the right-hand side of both (3.5) and (3.4). Computing the limit gives our result. Q.E.D.

In passing, let us note that the method of $\$ 1$ could have been used to prove the above result in a few lines since

$$
\begin{aligned}
\exp \left\{Q_{\lambda}^{1}[t, 0]+Q_{\lambda}^{2}[t, 0]\right\} & =\exp \left\{Q_{\lambda}^{1}[t, 0]\right\} \exp \left\{Q_{\lambda}^{2}[t, 0]\right\} \\
& =\exp \left\{Q_{\lambda}^{2}[t, 0]\right\} \exp \left\{Q_{\lambda}^{1}(t, 0]\right\}
\end{aligned}
$$

The reason for such a roundabout approach is partially revealed if we go back to $U_{\lambda}^{0}[t, 0]$ in (3.2) and assume that $\Delta t_{j}=1 / n, 1 \leqslant j \leqslant n$, so that

$$
U_{\lambda}^{0}[t, 0]=e^{-\lambda T} \sum_{n=0}^{\infty} \frac{(\lambda T)^{n}}{n !}\left[\prod_{j=1}^{n} \exp \left\{\frac{1}{n} A_{1}\left(\tau_{j}\right)\right\}\right]\left[\prod_{j=1}^{n} \exp \left\{\frac{1}{n} A_{2}\left(s_{j}\right)\right\}\right] .
$$

This certainly reminds us of the Trotter-Kato product formula which is fundamental to conventional studies on the addition of unbounded operators (cf. Chernoff [C]). The fact that Theorem 3.1 does not depend on the respective domains might give the impression of some underlying pathology; however, those unaware of the possibilities will be surprised (and inspired) by the work of Chernoff. In the most innocent of cases he shows that generalized additivity of unbounded operators may occur even if the domains only have the zero function in common. The exact relationship with the Trotter-Kato product formula requires the construction of a certain symmetric Banach space which is a generalization of Fock space and will be discussed at a later time. 
Let us recall that $L\left[\otimes_{\tau \in[0, T]}^{\alpha} B(\tau)\right]$ is the space of bounded operators on $\otimes_{\tau \in[0, T]}^{\alpha} B(\tau)$. Since $\lim _{\lambda \rightarrow \infty} U_{\lambda}^{i}[t, 0]=U^{i}[t, 0]=U^{i}[t, 0]$ exists when $\tau_{j} \in I_{1}, s_{j} \in I_{2}$, and since $L\left[\otimes_{\tau \in I}^{\alpha} B(\tau)\right]$ is complete, we conclude that $U^{i}[t, 0] \in L\left[\otimes_{\tau \in I}^{\alpha} B(\tau)\right]$, $i=0,1,2$. This result is quite nice since it implies that $\phi \otimes_{\tau \in I} B(\tau)$ (which is separable) may be used as our state space (film) for the study of the evolution equation

$$
\frac{\partial \phi(t)}{\partial t}=A(t) \phi(t), \quad \phi(0)=\phi
$$

This is also pleasing from a physical point of view since it may be interpreted to mean that the physical information about a system is already contained in a countable number of time slices. This result is also related to the sample path approach to random functions (cf. Loeve [Lo, pp. 500-501]).

It has recently been shown in two nonoverlapping examples by Goldstein [Go] and Dorroh [Dr] that the standard assumption of a dense common core for all times $t$ in the study of linear evolution equations cannot be expected to hold. They constructed unique solutions to the Cauchy problem (3.7) for $\phi$ in a dense subspace (in the conventional sense) with solutions depending continuously on the initial data, and in the Goldstein example, $\cap_{t} D(t)=\{0\}$, while in the Dorroh example $D(t) \cap$ $D(s)$ is a nowhere dense set of $s \neq t$. In our approach we assume no special relationship between respective domains.

We now obtain a number of other results in the foundations of linear evolution equations.

TheOREM 3.2. Suppose $\phi \in D\left[A^{N}(s)\right], 0 \leqslant s \leqslant t$. Then

$$
U[t, 0] \phi=\exp \left\{\int_{0}^{t} A(\tau) d \tau\right\} \phi .
$$

may be represented as

$$
U[t, 0] \phi=\sum_{k=0}^{N-1} \int_{0}^{t} d \tau_{k} \int_{0}^{\tau_{k}} d \tau_{k-1} \cdots \int_{0}^{\tau_{2}} d \tau_{1} A\left(\tau_{k}\right) \cdots A\left(\tau_{1}\right) \phi+R_{N}[t, 0] \phi,
$$

where $R_{N}[t, 0]$ satisfies

$$
\begin{aligned}
R_{N}[t, 0]= & \int_{0}^{1} d v(1-v)^{N} \exp \left\{v \int_{0}^{t} A(\tau) d \tau\right\} \\
& \times \int_{0}^{\tau} d \tau_{N} \cdots \int_{0}^{\tau_{2}} d \tau_{1} A\left(\tau_{n}\right) \cdots A\left(\tau_{1}\right) \phi .
\end{aligned}
$$

Proof. We know from Theorem 1.2 that

$$
Q^{z}[t, 0]=\int_{0}^{t} A^{z}(\tau) d \tau \text { and } Q[t, 0]=\int_{0}^{t} A(\tau) d \tau
$$


are both generators of strongly continuous semigroups of operators $s-\lim _{z \downarrow 0} Q^{z}[t, 0]$ $=Q[t, 0]$. Now

$$
U^{z}[t, 0] \phi=\sum_{k=0}^{N-1} \frac{\left(Q^{z}[t, 0]\right)^{k} \phi}{k !}+\int_{0}^{1} d v(1-v)^{N} \exp \left\{v Q^{z}[t .0]\right\} \frac{\left(Q^{=}[t .0]\right)^{Y}}{N !} \phi .
$$

(This result may be found in [HP, p. 354].)

Let us consider the case $k=2$. We have

$$
\frac{\left(Q^{z}[t, 0]\right)^{2}}{2 !} \phi=\frac{1}{2}\left[\int_{0}^{t} \int_{0}^{t} A^{z}\left(\tau_{1}\right) d \tau_{1} A^{z}\left(\tau_{2}\right) d \tau_{2}\right] \phi .
$$

The term on the right can be split up by considering $\tau_{1} \leqslant \tau_{2}, \tau_{1}>\tau_{2}$ :

$$
\frac{1}{2} \int_{0}^{t} \int_{0}^{\tau_{2}} A^{z}\left(\tau_{2}\right) A^{z}\left(\tau_{1}\right) d \tau_{1} d \tau_{2}+\frac{1}{2} \int_{0}^{t} \int_{\tau_{2}}^{t} A^{z}\left(\tau_{1}\right) A^{z}\left(\tau_{2}\right) d \tau_{2} d \tau_{1} .
$$

The region of integration is the square $0 \leqslant \tau_{1} \leqslant t, 0 \leqslant \tau_{2} \leqslant t$. We have divided it into two equal triangles: $0 \leqslant \tau_{1} \leqslant \tau_{2}, 0 \leqslant \tau_{2} \leqslant t$ and $\tau_{2} \leqslant \tau_{1} \leqslant t, 0 \leqslant \tau_{2} \leqslant t$. Let us rewrite the second integral as (interchange order of integration)

$$
\frac{1}{2} \int_{0}^{t} \int_{0}^{\tau_{1}} d \tau_{1} d \tau_{2} A^{z}\left(\tau_{1}\right) A^{z}\left(\tau_{2}\right)
$$

and replace $\tau_{1} \rightarrow \tau_{2}, \tau_{2} \rightarrow \tau_{1}$ so that

$$
\frac{\left(Q^{z}[t, 0]\right)^{2}}{2 !} \phi=\int_{0}^{t} \int_{0}^{\tau_{2}} A^{z}\left(\tau_{2}\right) A^{z}\left(\tau_{1}\right) d \tau_{1} d \tau_{2} .
$$

The result for all $k$ follows by induction; taking strong limits proves our theorem. Q.E.D.

In Theorem 3.1, let us replace $A_{2}(\tau)$ by $\varepsilon A_{2}(\tau), \varepsilon>0$, so that $U^{0}(t, 0]$ becomes

$$
U_{\varepsilon}^{0}[t, 0]=\exp \left\{\int_{0}^{t}\left[A_{1}(\tau)+\varepsilon A_{2}(\tau)\right]\right\} d \tau=U^{1}[t, 0] U_{\varepsilon}^{2}[t, 0] \text { a.s. }
$$

The following is a result of Feynman.

LEMMA 3.1.

$$
\left.\frac{d}{d \varepsilon} U_{\varepsilon}^{0}[t, 0]\right|_{\varepsilon=0}=\int_{0}^{t} U^{1}[t, \tau] A_{2}(\tau) U^{1}[\tau, 0] d \tau
$$

ProOF.

$$
\frac{d}{d \varepsilon} U_{\varepsilon}^{0}[t, 0]=U^{1}[t, 0] \frac{d}{d \varepsilon} U_{\varepsilon}^{2}[t, 0] \text { a.s. }
$$

since $\int_{0}^{t} \varepsilon A_{2}(\tau) d \tau=\varepsilon \int_{0}^{t} A_{2}(\tau) d \tau=\varepsilon Q_{2}[t, 0]$, and as $Q_{2}[t, 0]$ is a generator by Theorem 1.2, we have

$$
\frac{d}{d \varepsilon} U_{\varepsilon}^{2}[t, 0]=Q_{2}[t, 0] U_{\varepsilon}^{2}[t, 0]
$$


Hence

$$
\begin{aligned}
\left.\frac{d}{d \varepsilon} U_{\varepsilon}^{0}[t, 0]\right|_{\varepsilon=0} & =\left.U^{1}[t, 0] Q_{2}[t, 0] U_{\varepsilon}^{2}[t, 0]\right|_{\varepsilon=0} \text { a.s. } \\
& =\exp \left\{\int_{0}^{t} A_{1}(\bar{\tau}) \overline{d \tau}\right\} \int_{0}^{t} A_{2}(\tau) d \tau .
\end{aligned}
$$

We may split the right-hand side into regions with $\tau \leqslant \bar{\tau}$ and $\tau>\bar{\tau}$ to get

$$
\left.\frac{d}{d \varepsilon} U_{\varepsilon}^{0}[t, 0]\right|_{\varepsilon=0}=\int_{0}^{t} \exp \left\{\int_{\tau}^{t} A_{1}(\bar{\tau}) d \bar{\tau}\right\} A_{2}(\tau) \exp \left\{\int_{0}^{\tau} A_{1}(\bar{\tau}) d \bar{\tau}\right\} d \tau \text {. Q.E.D. }
$$

If we combine Theorem 3.2 with Lemma 3.1, we get

THEOREM 3.3. If $\phi \in D\left[A_{2}^{N}(s)\right], 0 \leqslant s \leqslant t$, then

$$
\begin{array}{r}
U^{0}[t, 0] \phi=\sum_{k=0}^{N-1} \int_{0}^{t} d \tau_{k} \cdots \int_{0}^{\tau_{2}} d \tau_{1} U^{1}\left[\tau_{k+1}, \tau_{k}\right] A_{2}\left(\tau_{k}\right) U^{1}\left[\tau_{k}, \tau_{k-1}\right] A_{2}\left(\tau_{k-1}\right) \\
\cdots U^{1}\left[\tau_{2}, \tau_{1}\right] A_{2}\left(\tau_{1}\right) U^{1}\left[\tau_{1}, \tau_{0}\right] \phi+\bar{R}_{N}[t, 0] \phi \text { a.s. }
\end{array}
$$

where $\tau_{0}=0$ and $\tau_{k+1}=t, 0 \leqslant k \leqslant N-1$, and

$$
\begin{aligned}
\bar{R}_{N}[t, 0] \phi= & \int_{0}^{1} d v(1-v)^{N} \exp \left\{v \int_{0}^{t} A_{2}(\tau) d \tau\right\} \int_{0}^{t} d \tau_{N} \\
& \times \int_{0}^{\tau_{2}} d \tau_{1} U^{1}\left[\tau_{N+1}, \tau_{N}\right] A_{2}\left(\tau_{n}\right) U^{1}\left[\tau_{N}, \tau_{N-1}\right] \cdots U^{1}\left[\tau_{1}, \tau_{0}\right]
\end{aligned}
$$

(3.13) and (3.14) were incorrectly written in [G4].

Theorem 3.3 is a generalization of the Feynman diagram method, in the sense that it does not depend on the existence of a propagator function for the evolution $U^{1}[t, 0]$, and we provide a remainder.

Let us now suppose that $A_{1}(\tau)$ is a selfadjoint generator of a unitary group and $A_{2}(\tau)$ does not depend on $\tau$, so that the time index simply tells us when it operates. Define $A_{I}(\tau)$ by (interaction representation)

$$
A_{I}(\tau)=\bar{U}^{1}[0, \tau] A_{2}(\tau) U^{1}[\tau, 0]
$$

and

$$
\bar{U}^{1}[0, \tau] U^{1}[\tau, 0]=U^{1}[\tau, 0] \bar{U}^{1}[0, \tau]=I .
$$

Under general conditions, it is not hard to see that $A_{I}(\tau)$ will be the generator of a contraction semigroup; however, in physics the case of interest is when $A_{I}(\tau)$ is a selfadjoint generator of a unitary group and $B(\tau)$ for each $\tau$ is a separable Hilbert space. This will be true if and only if $A_{2}(\tau)$ already has this property; replacing $(0, T)$ by $[-T, T]$ we have

$$
S[t,-t]=\exp \left\{\int_{-t}^{t} A_{I}(\tau) d \tau\right\}
$$

exists and is a well-defined unitary group. $S[t,-t]$ is known as the $S$-matrix in physics (field theory), and much effort has gone into identifying conditions under 
which it exists. Combining (3.17) with Theorem 3.2 we get

$$
S[t,-t] \phi=\sum_{k=0}^{N-1} \int_{-t}^{t} d \tau_{k} \cdots \int_{-t}^{\tau_{2}} d \tau_{1} A_{I}\left(\tau_{k}\right) \cdots A_{I}\left(\tau_{1}\right) \phi+R_{N}[t,-t] \phi .
$$

This is the standard form of the perturbation expansion of the $S$-matrix which is used for computation in quantum field theory (modulo manifest covariance). There is one major improvement, namely the inclusion of a remainder term after the $N$ th order computation.

Suppose we are given a general transition kernel $\tilde{\mathbf{K}}[x, t, y, s]$ which provides us with a transition of the system in position $y$ at time $s$ to position $x$ at time $t$. Let us further assume that this kernel is associated with the family of operators $\left\{\tilde{A}_{1}(\tau) \mid t \in\right.$ $[0, T]\}$ (all of this is assumed in the conventional sense) so that the equation

$$
\frac{\partial v}{\partial t}=\tilde{A}_{1}(t) v, \quad v(0)=v_{0},
$$

is satisfied by

$$
v(x, t)=\int_{R^{3}} \tilde{\mathbf{K}}[x, t, y, 0] v_{0}(y) d y=\tilde{U}^{1}[t, 0] v_{0} .
$$

If we replace the family $\left\{A_{1}(\tau) \mid \tau \in[0, T]\right\}$ by its time-ordered version $\left\{A_{1}(\tau) \mid \tau\right.$ $\in[0, T]\}$, a natural family of kernels $\mathbf{K}[x(t), t, x(s), s]$ is induced. In order to identify the exact relationship, let us return to (3.1):

$$
U_{n}^{1}[t, 0]=\exp \left\{\sum_{j=1}^{n} \int_{t_{j-1}}^{t_{j}} E\left[\tau_{j}, \tau\right] A_{1}(\tau) d \tau\right\} .
$$

From [G1] this may be written as

$$
\begin{aligned}
& U_{n}^{1}[t, 0]=\prod_{j=1}^{n} \exp \left\{\int_{t_{j-1}}^{t_{j}} E\left[\tau_{j}, \tau\right] A_{1}(\tau) d \tau\right\} \\
= & \prod_{j=1}^{n} \overline{[\underbrace{}_{0 \leqslant s<\tau_{j}} I_{s} \otimes\left(\tilde{U}^{1}\left[t_{j}, t_{j-1}\right]\right) \otimes(\underbrace{\left.\left.\bigotimes I_{s}\right)\right]}_{\tau_{j}<s \leqslant T}}
\end{aligned}
$$

$$
\begin{gathered}
=\prod_{j=1}^{n} \overline{\left[\bigotimes_{0 \leqslant s<\tau_{j}} I_{s} \otimes\left(\int_{R^{3}\left(t_{j-1}\right)} \tilde{\mathbf{K}}\left[x\left(t_{j}\right), t_{j}, x\left(t_{j-1}\right), t_{j-1}\right] d x\left(t_{j-1}\right)\right) \otimes(\underbrace{}_{\tau_{j}<s \leqslant T} I_{s})\right]} \\
\quad=\prod_{j=1}^{n} \int_{R^{3}\left(t_{j-1}\right)} d x\left(t_{j-1}\right) \mathbf{K}_{\tau_{j}}\left[x\left(t_{j}\right), t_{j}, x\left(t_{j-1}\right) t_{j-1}\right] .
\end{gathered}
$$

If we combine (3.22) with our definition of $U_{\lambda}^{1}[t, 0]$ (3.2), we have

$$
\begin{aligned}
U_{\lambda}^{1}[t, 0]= & e^{-\lambda T} \sum_{n=0}^{\infty} \frac{(\lambda T)^{n}}{n !} \prod_{j=1}^{n} \int_{R^{3}\left(t_{j-1}\right)} d x\left(t_{j-1}\right) \\
& \times \mathbf{K}_{\tau_{j}}\left[x\left(t_{j}\right), t_{j}, x\left(t_{j-1}\right), t_{j-1}\right] .
\end{aligned}
$$


Since the left-hand side of (3.23) exists as a bounded linear operator, we can view the right-hand side as an operator-valued (path) integral. From Theorem 2.1 we know that $\lim _{\lambda \rightarrow \infty} U_{\lambda}^{1}[t, 0]$ exists in the uniform operator topology, and we see that we may represent $U^{1}[t, 0]$ as an operator-valued path integral

$$
U^{1}[t, 0]=\int_{X} \mathbf{K}_{t}[x(t), t, x(0), p] D_{t}[x(0)] .
$$

We may now translate all our theorems in this section into statements about such path integrals. The important point is that a path integral exists whenever a transition kernel is available. The details will be presented elsewhere.

ACKNOWLEDGMENTS. While completing this work, the writer was partially supported by a Chancellor Postdoctoral Research Fellowship at the University of California, Berkeley. The encouragement and support of the Department of Mathematics is deeply appreciated. Special thanks are due to Professors William G. Bade and Paul R. Chernoff for much discussion and help. Professor Lee Lorch (York University, Toronto, Canada) was kind enough to explain some of the finer details associated with Borel summability methods.

\section{REFERENCES}

[C] P. R. Chernoff, Product formulas, nonlinear semigroups, and the addition of unbounded operators, Mem. Amer. Math. Soc. No. 140 (1970).

[Dr] J. R. Dorroh, A linear evolution equation without a common dense core for the generators, J. Differential Equations 31 (1979).

[F1] R. P. Feynman, Space-time approach to non-relativisitic quantum mechanics, Rev. Modern Phys. 20 (1948)

[F2] __ An operator calculus having applications in quantum electrodynamics, Phys. Rev. 84 (1951).

[G1] T. L. Gill, Time-ordered operators. I: Foundations for an alternate view of reality, Trans. Amer. Math. Soc. 266 (1981), 161-181.

[G2] __ Infinite tensor products of Banach spaces. I, J. Funct. Anal. 30 (1978).

[G3] New perspectives in time-ordered operators and divergencies. I, Hadronic J. 3 (1980), $1597-1621$.

[G4] New perspectives in time-ordered operators and divergencies. II, Hadronic J. 3 (1980), 1597-1621.

[Go] J. A. Goldstein, On the absence of necessary conditions for linear evolution operators, Proc. Amer. Math. Soc. 44 (1977).

[Gr] A. Grothendieck, Produits tensoriels topologiques et espaces nucléaires, Mem. Amer. Math. Soc. No. 16 (1935).

[HP] E. Hille and R. S. Phillips, Functional analysis and semigroups, Amer. Math. Soc. Colloq. Publ., vol. 31, Amer. Math. Soc., Providence, R. I., 1957.

[H] J. R. Holub, Hilbertian operators and reflexive tensor products, Pacific J. Math. 36 (1971).

[Lo] M. Loeve, Probability theory, 3rd ed., Van Nostrand, New York, 1963.

[S] R. Shatten, $A$ theory of cross-spaces, Princeton Univ. Press, Princeton, N.J., 1950.

Department of Mathematics, University of California, Berkeley, California 94720

Current address: Department of Mathematics, Howard University, Washington, D.C. 20059 